\title{
$X$.
}

Aus der Abtheilnng für Ohrenkranke der Kgl. Charité in Berlin (dirigirender Arzt: Geh. Med.-Rath Prof. Dr. Trautmann).

\section{Zur Function der Bogengänge.}

Von

Stabsarzt Dr. Stenger,

z. Z. Assistenten der Klinik.

Der mannigfachsten Thierversuche hat es bedurft, bis aber die Function der Bogengänge auch nur einigermaassen Klarheit gewonnen war. Hatten sich die älteren Autoren auf Grund schematischer Deductionen oder auf vergleichend anatomische Studien hin Hypothesen über die Function der einzelnen Theile des inneren Ohres gebildet, so war es Flourens, der an der Hand des Thierexperimentes den richtigen Weg zeigte. Er zerstörte experimentell die einzelnen Organe isolirt und fand bei Durchschneidung der Bogengänge eigenthümliche Erscheinungen von Gleichgewichtsstörungen, die ihn zu weiteren Versuchen anregten. Seitdem unternahmen es zahlreiche Forscher, auf dem gewiesenen Weg des Thierexperiments Gewissheit ubber die Funetion der Bogengänge zu erlangen. Das Resultat war ein sehr verschiedenes. Mit Ruteksicht auf die tuberaus grosse Schwierigkeit des exacten Experiments blieben der Speculation immer noch weite Bahnen offen. Flourens(1) schon fand, dass die Zerstörung der Bogengänge das Gehör nicht vernichtet, dass also der Nervus acusticus kein einfacher Nerv, sondern aus einem mit aeustiseher Function begabten Nerven für die Schnecke und dem mit Bewegungsfunctionen begabten Vestibularnerven zusammengesetzter Nerv sei. Verletzungen der Bogengänge fuhren also nur zweckmässig auftretende Bewegungen in der Richtung des verletzten Ganges herbei, ohne Beeinträchtigung des Gehörs.

Löwenberg (2) fand als Resultat seiner Untersuchungen:

1. Die nach Dursehschneidung der Semicircularcanäle des Ohres auftretende Bewegungsstörungen hängen nur von dieser 
Verletzung und nicht von etwaiger Mitverletzung von Hirntheilen $a b$.

2. Das von Prof. Czermak bei seinen Experimenten beobachtete Erbrechen hängt von Mitverletzung des Kleinhirns ab.

3. Die Bewegungsstörungen sind Folge von Reizung der häutigen Canäle und nicht von Lähmung derselben.

4. Die Reizung der häutigen Canäle ruft die krampfhaften Bewegungen auf reflectorischen Wege hervor, ohne jede Betheiligung des Bewusstseins. Das Bewusstsein spielt bei diesen Vorgängen nur insofern eine Rolle, als es durch Hervorrufen willkturlicher Bewegungen der Thiere zu neuen Reizungen der häutigen Canäle Anlass giebt.

5. Die Uebertragung dieser reflectorischen Erregung von den Nerven der häntigen Semicircularcanäle auf die motorischen Nerven findet im Thalamus statt.

Goltz (3) sieht von der Frage, ob die Bogengänge uberhaupt Gehörorgane seien, ganz ab und bezeichnet sie als eine Vorrichtung, welche der Erhaltung des Gleichgewichts dient. Sie sind sozusagen Sinnesorgane fur das Gleichgewicht des Kopfes und mittelbar des ganzen Körpers.

Mach (4) hält die halbeirkelförmigen Canäle für ein Orgài, welches zur Empfindung der Kopfbewegungen dient. (Organ der Bewegungsempfindung, Organ der Beschleunigungsempfindung.)

Breuer und Brown (5 u. 6) schliessen sich im Ganzen Machs Ansicht an, Breuer mit der Erweiterung, dass speciell die Otolithen zur Wahrnehmung der Körperbewegung dienten. Come B row n meint, dass die halbcirkelförmigen Canäle gepaarte Organe seien, und dass jeder Canal nur der Drehungsempfindung in einer einzigen Ebene entspricht.

Cyon (7) erkennt die akustische Bedeutung der halbeirkelförmigen Canäle nicht an und bezeichnet dieselben als Sinnesorgane zur Bestimmung der 3 Dimensionen.

Laborde (8) bezeichnet dic Bogengänge als einen Empfindungs- und Bewegungsapparat, der mit dem Gehörorgan verbunden ist, und die durch Schalleindrücke hervorgerufenen Bewegungen des Kopfes und Rumpfes leitet.

Viguiers und Delage (9 u. 10) schliessen aus Beobachtungen an Thieren und Mensehen auf ein besonderes Sinnesorgan der Orientirung im Raum und verlegen den Sitz desselben in die halbeirkelförmigen Canäle. 
Ewald (11) kommt auf Grund zahlreicher Versuche zu der Ansicht, dass sich das Endorgan des Nervus acustieus physiologisch aus folgenden Apparaten zusammensetzt:

1. Dem Tonuslabyrinth, das einen beständigen Einfluss anf die Nervenbahnen ausübe. Es besteht aus dem Bogengangsapparat (Goltz'sehen Sinnesorgan) und dem Otolithenapparat (Maculae acusticae).

2. Dem Hörlabyrinth. Der Bogengangsapparat ist demnach vom Höract getrennt.

Böttcher (12) gewinnt auf Grund seiner Untersuchungen die Ueberzeugung, dass die bei Verletzung der Bogengänge anftretenden Reitbahnbewegungen, Ueberschwanken nach vorn und hinten, und die anderen unregelmässigen Gleichgewichtsstörungen des Rumpfes, ebenso wie die Verdrehung des Kopfes und die sich daran knüpfenden Rollbewegungen in Veränderungen des Gehirns ihren Grund haben. Ueber die Art der nach Verletzung der Bogengänge eintretenden localen Veränderungen im Hirn müsse das Mikroskop Aufschluss geben.

Berthold (13) theilt den Bogengängen die Function zu, die Coordination der Bewegungen auf dem Wege des Reflexes zu vermitteln. Sie leisten diese Arbeit in Verbindung mit 2 Sinnesorganen, mit dem Gesichts- und dem Gefuhlssinn. Ob die Bogengänge mit dem Höract in Verbindung stehen, lässt Berthold freigestellt.

Baginski (14) theilt Versuche mit, aus denen er sehliesst, dass die isolirte Verletzung der Bogengänge ohne Rückwirkung auf das Gehirn nicht möglich sei, und dass der Zeitpunkt gekommen sei, die Lehre von dem statischen Sinne in den Bogengängen ein für alle Male aufugeben. Nichts berechtige, aus Veränderungen, die sich nach der Bogengangsverletzung an Thieren zeigen, Sehlïsse zu machen auf die Funetion der Bogengänge, nachdem er den bestimmten Nachweis geführt habe, dass eine Verletzung der Bogengänge sowohl bei Säugethieren als auch bei Tauben jedesmal eine Verletzung des Gehirns involvirt.

Auch v. Bergmann (15) steht ganz unter dem Einfluss der Böttcher'schen Versuche und glaubt, die nach Verletzung der Bogengänge auftretenden Erscheinungen auf eine gleichzeitige Verletzung des Gehirns schieben zu mussen. Gleichzeitig beruft er sich auf die Resultate der Untersuchungen $\mathrm{C}$ urs ch mann's (16), der nach Zerstörung aller häutigen Canäle keinen völligen Verlust des Körpergleichgewiohts gesehen hat und nach einfachen Durch- 
schneidungen schon innerhalb einer Woche die Symptome schwinden sah, ein Factum, das sich mit der den Bogengängen ertheilten Rolle als Organ des Gleichgewichts nicht gut vereinigen lässt.

v. Bergma n n meint, da die klinischen Thatsachen mehr für Böttcher als die anderen Forscher sprechen, so sind Schwindelund Gleichgewichtsstörungen, Reitbahngang und Umfallen nach der kranken Seite weniger als Zeichen einer das Labyrinth durchsetzenden Knochenfissur, denn als Symptom einer gleichzeitigen Hirnläsion anzusehen. Einen extremen Standpunkt nimmt Br tick n er (17) ein, indem er die halbzirkelförmigen Canäle als Sinnesorgan fur das Gleichgewicht des Kopfes und mittelbar des ganzen Körpers vollständig aussehliesst und dieselben nur als zum Gehöract dienend ansieht und annimmt, dass von den 3 Bogengängen in ihrer bestehenden Combination zum Hören leiser Geräusche immer ein horizontaler liegender Bogengang für jede der verschiedenen Hauptstellungen des Kopfes nothwendig sei.

$\mathrm{Zu}$ einem sicheren und befriedigenden Resultate haben demnach die Thierversuche bisher nicht geftuht. Seitdem es der Operationstechnik gelungen ist, das am lebenden Menschen bisher unerreichbare Labyrinth uns zugänglich za machen, mehren sich auch die Beobachtungen am Krankenbett uber die Verletzungen der Bogengänge und sicherlich sind diese nothwendig, um zur Ergänzung und fussend auf den Resultaten des Thierexperiments uns in letzter Linie volle Aufklärung über die Function der einzelnen Theile des Labyrinthes zu geben.

Aus der Menge der im Laufe der Jahre operirten und Analoges bietenden Falle, nehme ich nur die im letzten Jahre beobachteten Fälle mit Erkrankung bezw. Verletzung der horizontalen Bogengänge heraus zur Erläuterung der bei uns danach beobachteten Erscheinungen.

1. Johann G., 57 jähriger Arbeiter. Aufgenommen am 2. September 1898. Rechts alte Antrumwunde, nocb bestehende eitrige Secretion aus dem änsseren Gehörgang. Flüstersprache rechts nicht gehört.

8. November 1899. Radicaloperation. Caries der medialen Autrumwand und am Tegmen tympani. Beim Entfernen der cariösen Massen wird der horizontale Bogengang eröffnet.

Nach der Operation kein auffallend verstärktes Erbrechen. Klagen über Kopfschmerzen und Flimmern vor den Augen.

12. November. Puls andauernd regelmässig. Keine Temperaturerhöhung. Augenbewegungen frei, kein Nystagmus. Kann sich im Bett aufsetzen, doch tritt heftiges Schwindelgefühl auf. Die Gegenstände im Zimmer drehen sich vor seinen Augen, er weiss nicht, was unten und oben ist.

15. November. Erster Verbandwecbsel. Andauerndes Schwindelgefühl Beim Liegen auf der kranken Seite besteht das Gefühl, als versänke der Be- 
treffende. Beim Aufrichten drehen sich die Gegenstände im Zimmer von rechts nach links. Dabei besteht die Neigung nach reebts zu fallen. Kein Nystagmus. Bei Berührung des eröffneten Bogengangs starkes Schwindelgefübl und unregelmässige Angenbewegungen.

18. November. Patient kann aufstehen und einzelne Schritte gehen, doch fallt er dabei nach rechts und hinten, bei Romberg starkes Schwanken und schliesslich Umfallen nach der rechten Seite. Beim Gehen mit offenen Augen besteht das Bestreben, nach rechts abzuweichen, deutlicher ist dies beim Gehen mit geschlossenen Augen. Sehen auf die belebte Strasse macht ihn ganz verwirrt. Die Lente und Fahrzeuge erscheinen ihm verzerrt. Er kann nicht lange hinseben, ohne schwindlig zu werden. Wendungen werden unsicher und sehr vorsichtig ausgeführt, bei geschlossenen Augen unter stărkerem Schwanken. Sensibilität erscheint auf der ganzen rechten Körperseite etwas gegen links herabgesetzt. Nadelstiche werden rechts undeutlicher als links empfunden. Gefübl für Kälte und Wärme beiderseits gleich. Grobe Muskelkraft beiderseits nicht herabgesetzt. Arm- und Beinbewegungen werden regelrecht ausgeführt. Versuch, mit der linken Ferse das rechte Knie bei geschlossenen Augen schnell zu berahren, wird mit ziemlicher Sicherheit und ohne Nebenbewegungen ausgefürt. Patellarreflexe erhalten, nicht verstärkt.

25. November. Kein Nystagmus, Gang noch unsicher, besonders im Dunkeln. Beim Aufrichten im Bett schwankt er noch bin und her.

28. November. Schwindelgefuhl ist nicht mehr so stark, weder beim Aufrichten im Bett, noch beim Aufstehen. Der Gang ist etwas unsicher, doch besteht noch Schwanken. Er geht breitbeinig und langsam, den Blick zum Boden gesenkt. Bei Wendungen ist die Wendung vach rechts erschwert, langsam und unsicher. Beim Geradeausgehen kein Abweichen mehr. Beim Blick anf die Strasse kann er alles erkennev, nur bei längerem Hinsehen vermischen sich die Bilder. Grössere Buchstaben auf Ladenschildern drehen sich beim Versuch zum Lesen. Armbewegungen sind frei. Seusibilität ist nicht gestört. Bei längerer Beobachtung und eintretender Ermüdung werden die Schwindelerscheinungen wieder stärker, besonders bei geschlossenen Augen.

30. November. Keine Kopf'schmerzen. Seit gestern Ohrensausen rechts, das fortwährend besteht und theils in andauerndem Klingeln, theils in anhaltendem Poltern besteht. Beide Geräusche werden für sich getrennt gehört. Der Schlaf ist dadurch nicht gestört, doch träumt er sehr lebhaft und schlät nach dem Aufwachen sehr sehwer wieder ein.

12. December. Das Schwindelgefühl lässt nach. Die subjectiven Geräusche sind noch unvermindert heftig. Der Gang ist ziemlich sicher. Bei geschlossenen Augen werden Kehrtwendungen nach rechts noch sehr unsicher ausgeführt.

18. Januar. Es besteht nur noch leichtes Schwindejgefühl im Dunkeln.

27. Mărz. Geheilt entlassen. Die subjectiven Geräusche bestehen noch in geringem Grade. Schwindelgefühl besteht nicht mehr, auch im Dunkeln nicht. Bei schnellen Wendungen nach rechts lässt sich noch eine gewisse Unsicherheit erkennen.

Mitte Mai bei einer erneuten Vorstellung besteht kein Schwindelgefühl mehr, auch die subjectiven Gerăusche haben nachgelassen. Flüstersprache rechts nicht gehört.

11. Friedrich K., 25 Jahre alt, Schumacher. Aufgenommen den 27. Januar 1899. Wegen cbronischer Mittelohreiterung beiderseits auswärts radical operirt. Rechts verheilte Radicaloperationswunde, links Wundhöhle mit schmierigen Granulationen.

Subjective Klagen über Sausen im linken Ohr. Keine Kopfschmerzen, kein Schwindel. Romberg negativ. Kein Nystagmus. Flüstersprache links nicht gehört.

3. Februar 1898. Radicaln a ch operation links. Erweiterung der Wundhöhle. Entfernung des unteren Blatts der oberen Gehörgangswand, der hintere Theil des horizontalen Bogengangs erweist sich cariös. Das Lumen ist sichtbar. 
Nach der Operation ziemlich heftiges, andauerndes Erbrechen. Klagen über Schwindel und Uebelkeit.

8. Februar. Beim ersten Verbandwechsel wurde der raub aussehende borizontale Bogengang mit der Trephine in fast seiner ganzen Ausdebnung eröffnet und geglättet.

9. Februar. Subjectives lebhaftes Schwindelgefühl beim Aufrichten im Bett. Starkes Flimmern vor den Augen. Horizontaler Nystagmus in geringem Grade bei Bewegungen nach der gesunden Seite. Kein Erbrechen. Puls regelmässig, kräftig, leicht beschleunigt.

13. Februar. Nur geringes Schwindelgefühl. Patient geht breitbeinig, sehr vorsichtig, mit zu Boden gesenktem Blick. Wendungen werden vorsichtig und langsam ausgeführt. Bei Wendungen nach der kranken Seite stärkeres Schwanken. Bei geschlossenen Augen geht Patient auffallend unsicher, mit der Neigung, nach der linken Seite abzuweichen. Bewegungen des Kopfes nach der linken Seite setzt er erbeblichen Widerstand gegenüber, nach rechts nicht, bei Neigung des Kopfes nach links tritt Schwindelgefühl auf.

10. Februar. Gang heate sehr unsicher, mit Abweichen nach der linken Seite. Klagt über heftiges Herzklopien nach Bewegungen. Puls 72 nach mehrmaligem Hin- und Hergehen 120. Nystagmus nach rechts und links. Vor beiden Augen lebhaftes Flimmern, besonders bei Lageveränderung, so beim Aufstehen aus dem Bett und Umbergehen. Bei Seitwärtsdreheu des Kopfes kein Schwindel.

22. Februar. Noch lebhaftes Flimmern vor den Augen. Augenhintergrund normal. Nystagmus nur noch gering, nach rechts. Bei Bettlage Schwindel, besonders bei Lage auf der linken Seite, er hat dann das Getühl, als versảnke das Bett. Beim Gehen fühlt er sich noch immer unsicher, als wenn er betrunken wäre. Er geht noch breitbeinig, vorsichtig, mit Blick auf den Boden geheftet; Kehrtwendungen unsicher, bei geschlossenen Augen verbunden mit starkem Schwanken. Festes Auftreten auf den Fussboden mit dem linken Bein ruft schmerzhaftes Sausen im linken Obr hervor. Beim Berühren des Bogengangs kein Schwindel.

8. März. Kein Nystagmus. Gang sicherer.

22. März. Seit gestern lautes Sausen, schrilles Pfeifen im linken Ohr. Beim Liegen auf dem linken $\mathrm{Ohr}$ ist das Sausen nicht so stark, doch tritt dann noch Sehwindel auf. Bei geschlossenen Augen ist der Gang noch schr unsicher.

9. Mai. Die subjectiven Geräusche sind nicht mehr so laut. Schwindelgefühl besteht nur nach körperlicher Anstrengang und beim Gehen im Dunkeln. Kehrtwendungen werden sicher ausgeführt.

1. Juli. Keine subjectiven Geräusche mehr. Schwindelgefübl nur noch wenig beim Bücken und im Dunkeln. Flüstersprache links nicht gehört.

III. Emil M., 57 Jahre alt, Klempner. Aufgenommen am 7. Februar 1899. Seit 10 Jahren Ohreiterung rechts, seit Januar 1899 Kopfschmerzen und starkes Schwindelgefubl. Augenbewegungen frei. Kein Nystagmus. Flüstersprache rechts nicht gehört. Urin enthält Zucker.

$$
\mathrm{WR}+\mathrm{RiR}-\text {. }
$$

Beim Gehen subjectives Schwindelgefühl und Schwanken. Keine Lähmungserscheinungen, keine Ataxie.

9. Februar 1899. Radicaloperation rechts. Der horizontale Bogengang ist an seiner medialen Seite cariôs, wird abgemeisselt und mit der Trephine geglättet.

10. Februar. Klagen über Schmerzen im Kopf, Schwindel beim Aufrichten und Versuch nach der rechten Seite sich zu legen. Lebhafter Nystagmus nach der linken Seite, auch nach oben. Nur einmaliges, leichtes Erbrechen.

13. Februar. Lebhafter Nystagmus. Gegenstände im Zimmer drehen sich von rechts nach links. Beim Liegen nur geringes Schwindelgefühl. Kein Erbrechen.

16. Februar. Patient ausser Bett. Beim Gehen mit offenen Augen kein Schwindel. Wendungen nach rechts noch unsicher ausgeführt. Bei Romberg 
noch leichtes Schwanken. Bei Drehungen des Kopfes kein Schwindel, auch nicht beim Blick auf die belebte Strasse. Bei Blick nach links leichter Nystagmus. Grobe Muskelkraft erhalten. Im Gebrauch der Muskeln lässt sich keine Unregelmässigkeit nachweisen.

22. Februar. Schwindelgefühl noch beim Aufstehen und nach längerem und schnellerem Umhergeben. Auftreten mit beiden Fussen gleich sicher. Der Gang ist noch breitbeinig, dabei ist der Blick zu Boden gesenkt. Kein Nystagmus.

1. März. Seit gestern Bestehen subjectiver Geräusche im linken Ohr, Pfeifen und Klingen. Schwindelgefähl nur noch in geringem Grade.

13. März. Wegen Diabetes mellitus zur inneren Station verlegt. Schwindelgefübl und subjectives Sausen bestehen noch.

11. A pril. Wundhöble fest epidermisirt. Diabetes gebessert. Subjective Geräusche bestehen noch. Schwindelgefühl tritt nach schnellerem Gehen und beim Bücken noch auf. Flüstersprache rechts nicht gehört. (W R +.) Stimmgabeln durch Luft nicht gehört.

22. Juli. Kein Nystagmus, Romberg negativ. Keine subjectiven Geräusche. Schwindelgefübl nur nach angestrengter Arbeit (Patient versieht seinen Beruf als Monteur). Allgemeinbefinden gut.

25. October. Im Allgemeinen kein Schwindel. Im Dunkeln Gefühl von Unsicherheit. Vor 14 Tagen hat Patient einen in den Bahnhof einfahrenden Eisenbahnzug scharf angesehen, um sich ein Abtheil zu sichern, dabei ist er plötzlich so schwíndlig geworden, dass er hinfiel, ohne Bewusstseinsstörung. In belebten Strassen geht er im Gefühl der Unsicherheit dicht an den Häusern entlang, während er freie Plätze vollständig sicher überschreiten kann.

IV. Elise L., 24 Jahre alt, Arbeiterin. Aufgenommen den 6. Mărz 1899. 28. Juni 1694 wegen linksseitiger chronischer Mittelohreiterung Kadicaloperation links.

13. November 1894. Wunde geheilt.

Seit Weibnacbten 1895 ist die Wunde wieder aufgebrochen und eitert. Gleichzeitig ist Schwindelgefühl aufgetreten. Jetzige Klagen Kopfscbmerzen und Schwindelgefühl. Augenbewegungen sind frei. Kein Nystagmus. Pupillen gleichweit, reagiren. Links alte Radicaloperationswunde. In der Gegend des Facialiswulstes und des horizontalen Bogengangs findet sich eine dicke Granulationsmasse, in deren Tiefe man mit der Sonde auf rauhen Knochen stösst. Flüsterspracbe lin $\mathrm{ks} 10 \mathrm{~cm}$.

7. März. Radicaloperation links. Der mittlere Theil des horizontalen Bogengangs ist cariös und wird mit dem Meissel entfernt und mit der Trephine gegiättet, so dass man das Lumen gut übersehen kann.

8. März. Nur geringes Erbrechen. Heftiges Schwindelgefühl beim geringsten Versucb, sich aufzurichten oder im Bett umzudrehen. Starkes Flimmern vor den Augen. Die Gegenstände im Zimmer drehen sich von links nach rechts. Kein Nystagmus,

16. Januar. Geringer Nystagmus nach rechts. Schwindelgefühl beim Aufrichten und Gehen, taumelt nach rechts. Beim Sitzen auf einem Stuhl Schwindel nach links, besonders bei geschlossenen Augen. Beim Senken des Kopfes nach links stärkerer Schwindel, nach rechts nicht. Schwindelgefühl äberhaupt Morgens stärker als Abends. Beim Verbandweehsel kein stärkerer Schwindel.

23. März. Kein Nystagmus. Schwindelgefühl nur Morgens beim Aufstehen. Beim Ansehen von sich bewegenden Gegenständev, z. B. auf fliessendes Wasser, wird sie leichter schwindlig. Bei Wendungen starkes Schwanken nach links, ebenfalls bei Romberg. Beim Senken des Kopfes nach links noch stärkerer Schwindel. Beim Drehen des Kopfes ebenfalls. Die Gegenstânde dreben sich von links nach rechts. Gang breitbeinig, langsam mit zu Boden gesenktem Blick. Bei aufwärts gerichteten Augen starkes Schwanken und Abweichen nach links, Im linken Ohr laute subjective Gerâusche, die wie Leierkastenspielen' 'geschildert werden und Nachts den Schlaf stören.

23. April. Das Schwindelgefubl ist nur noch in geringem Grade vor- 
handen, tritt auf nach körperlicher Anstrengung und im Dunkeln. Die subjectiven Gerăusche besteben noch ziemlich unvermindert stark.

22. Mai. Die subjectiven Geräusche bestehen nicht mehr. Noch leichtes Schwindelgefühl im Dunkeln. Gang sicher; ohne Schwanken und Abweichen nach der Seite. Flüstersprache links $10 \mathrm{~cm}$.

V. Frau G., 40 Jabre alt, Zimmermannsfrau. Aufgenommen am 19. Juni 1899.

Seit 4 Jahren Ohreiterung beiderseitig. Seit Februar 1899 stărkere Eiterung, Kopfschmerzen und zunebmendes Schwindelgefübl. Bei Romberg kein Schwanken, kein Nystagmus, Augenhintergrund normal. Flustersprache links $2^{1 / 2} \mathbf{m}$, rechts $1^{1 / 2} \mathrm{~m}$. W $\mathrm{R}+$. Ri bds. -. Stimmgabeln von 60 abwärts nicht gehört, die höchsten Töne werden gehört. Links: Defect im unteren Abschnitt. Rechts: Fast Totaldefect, oberer kleinerer Rest mit Hammer, stark gewulstete Paukenschleimhaut.

21. Juni 1899. Radicaloperation rechts. Caries des horizontalen Bogengangs und des Tegmen antri. Der Bogengang wird mit der Trephine abgetragen und geglăttet.

22. Juni. Heftiges Erbrechen. Starkes Schwindelgefühl bei Bewegungsversuchen, kann nur auf der linken Seite liegen und sich nicht allein aufrichten, fällt sofort hintenüber. Kein Nystagmus.

24. Juni. Kein Erbrechen mehr. Bei richtiger Bettlage besteht das Gefühl, als drehen sich die Gegenstände im Zimmer und $z$ war von links nach rechts. Beim Versuch, sich aufzurichten, fallt sie nach hinten über.

27. Juni. Kein Nystagmus. Beim Versuch, Patientin hinzustellen, fâllt sie sofort in sich zusammen unter lebhaften Ármbewegungen. Kann noch nicht aufrecht sitzen. Beim Verbandwechsel stärkerer Schwindel.

2. Juli. Patientin kann heute zwar stehon, fällt aber sofort nach hinten und rechts um. Bei Romberg sofortiges Fallen nach rechts und hinten. Bei Drehungen des Kopfes starker Schwindel derart, dass sie durch Ármbewegungen das Gleichgewicht zu erhalten sucht.

5. Juli. Heute leichter Nystagmus nach links. Beim Liegen auf der linken Seite kein Schwindel, auf der rechten Schwindel und Gefühl des Fallens.

Beim Sitzen Schwindel, als fielen die Gegenstände nach rechts. Bei Drehungen des Kopfes um die senkrechte Achse ist dies Gefühl stärker. Beim Stehen mit offenen Augen leichtes Schwanken nach rechts und hinten, bei geschlossenen Augen starkes Schwanken mit Ausstrecken der Arme. Gang sehr vorsichtig, langsam mit gespreizten Beinen, keine Ataxie. Die Augen sind fest auf den Boden gerichtet, bei Augenschluss starkes Schwanken. Nach melreren Schritten plötalich Fallen nach binten und rechts. Beim Blick auf die belebte Strasse lebhafter Schwindel und starkes Flimmern vor den Augen.

9. Juli. Bei ruhiger Bettlage am Tage kein Schwindelgefühl. Bei Neigung des Kopfes nach rechts starker Schwindel und Drehen der Gegenstände nach rechts. Beim Vornüberneigen des Kopfes kein Schwindel, mehr aber bei Hintenüberneigen. Bei zunehmender Dunkelbeit ist der Schwindel überhaupt stärker, besonders wenn Patientin während des Nachts aufwacht. Sie kann sich dann zunächst gar nicht orientiren.

17. Juli. Patientin geht nur mit Unterstützung, muss auch beim Verbinden gestätzt werden. Keine subjectiven Geräusche. Nystagmus nur angedentet nach links Lässt man beim Sehen einen Gegenstand fixiren, so wird Patientin sofort stark schwindlig und will nach der rechten Seite umfallen.

20. Juli. Seit heute lautes Sausen im rechten $\mathrm{Ohr}$, wie Wasserrauschen. Patientin ist in der Nacht aufgewacht.

31. Juli. Patientin geht jetat allein ohne Unterstutzung. Der Gang ist breitbeinig, langsam und vorsichtig mit fest auf den Boden gerichtetem Blick. Kehrtwendungen werden ausserst vorsichtig ausgefuhrt. Dabei fallt Patientin noch meist nach hinten über, besonders bei Wendungen rach rechts. Beim Gang geradeaus Abweichen nach rechts, besonders bei geschlossenen Augen. 
15. August. Die subjectiven Geräusche bestehen noch fort. Nystagmus nicht mehr nachweisbar. Beim Gehen kein Schwanken; beim Stehen mit offenen Augen erst nach längerer Zeit Schwanken; bei geschlossenen Augen sofort. Bei Kehrtwendungen noch starkes Schwanken.

13. September. Die subjectiven Geräusche sind nicht mehr so lant. Im Uebrigen ist eine Besserung nicht eingetreten.

28. September. An der Labyrinthwand findet sich eine stark granulirende Stelle, die mit der Sonde sich ranh anfühlt. Abkratzen derselben mit dem scharfen Löffel.

14. October. Patientin wird gebessert entlassen. Die subjectiven Geräusche bestehen nicht mehr. Beim Gehen kein Schwanken. Gang noch vorsichtig und langsam. Schwindelgefühl nach längerem Gehen nur im Dunkeln. Flüstersprache $40 \mathrm{~cm}$ gehört.

VI. Emilie T., 24 Jahre alt, Näherin. Aufgenommen am 11. März 1897.

Seit frühester Jugend nach Diphtherie Ohreneiterung links. Seit einigen Wochen Schwindel, besonders Morgens und Abends, zeitweise Kopfschmerzen und zunehmende Schwerhörigkeit. Flästersprache links dicht vor dem Ohr. Romberg -. Augenhintergrund normal. Links: Totaldefect, hinten unten starke Granulationsbildung.

23. März. Radicaloperation. Recessus und Paukenhöhle voll Granulationen, Hammer, Amboss und Steigbügel fest in Granulationen eingeheilt. Steigbugel wird mit entfernt. Horizontaler Bogengang cariös, wird mit dem Meissel eröffnet und geglättet.

25. März. Puls regelmässig. Nur geringes Erbrechen. Kein Nystagmus. Starkes Schwindelgefühl. Unvermögen sich aufzurichten.

29. März. Kein Nystagmus, Kann sich im Bett aufsetzen, doch unter starkem Schwindelgefühl. Beim Gehen starkes Schwanken, besonders bei Kehrtwendungen.

2. Mai. Gebessert entlassen. Beim Gehen und Bücken noch Schwindel. Flüstersprache $20 \mathrm{~cm}$.

25. Mai. Wunde geheilt. Schwindelgefühl besteht noch in geringem Grade.

VII. Arthur S., 5 Jahre alt. Aufgenommen am 10. April 1899.

Seit dem 1. Lebensjabre rechtsseitige andauernde Eiterung aus unbekannter Ursache. Flüstersprache rechts dicht am Ohr. Kein Schwindel. Rechts fast Totaldefect, stark granulirende Paukenschleimhaut. Radicaloperation am 31. Mai 1899. Antrum erfüllt mit röthlich gelbem, glänzenden Brei und grossen Granulationen. Mediale Antrumwand cariös, ebenfalls horizontaler Bogengang, Paukenhöhle und Recessus mit dicken Granulationen ausgefüllt. Der cariöse Bogengang wird mit der Trephive geglättet.

2. Juni 1899. Nur geringes Erbrechen. Puls regelmässig, kräftig. Keine Schwindelerscheinungen. Patient sitzt aufrecht im Bett, ist sehr munter. Kein Nystagmus.

5. Juni. I. Verbandwechsel. Kein Nystagmus.

9. Juni. Keine Anzeichen von Schwindel. Patient geht ohne zu schwanken, auch mit geschlossenen Augen, ist sehr munter und spielt mit den anderen Kindern, steht auf einem Bein, ohne hinzufallen. Bei Kehrtwendungen kein Schwauken.

10. August. Keine Anzeichen von. Schwindel. Flüstersprache dicht am Ohr gehört. Allgemeines Wohlbefinden. Wunde noch nicht vollkommen verheilt. Gebessert entlassen.

VIII. Erna S., 3 Jahre alt. Aufgenommen am 14. August 1899.

Seit frähester Jugend aus unbekannter Ursache Ohreiterung rechts. Deshalb im April 1899 auswärts Eröffinung des Antrum. Hinter dem rechten Ohr nicht verheilte Antrumwunde, in deren Tiefe man rawhen Knochen fühlt, Aus dem rechten Obr profuse Eiterung. Flüstersprache rechts nicht gehört. 20. August 1899. Radicaloperation. Antrum und Paukenböble mit dicken Granulationen ausgefüllt. Der horizontale Bogengang ist cariös, und wird mit dem Meissel eröffnet und geglättet.

22. August. Kein Erbrechen. Wohlbefinden. Kein Schwindel. 
25. August. Kein Nystagmus. Patientin ist ausser Bett, spielt mit den anderen Kindern und zeigt in keiner Weise Erscheinungen von Schwindel.

18. October. Wunde fast vollkommen verheilt. Flästersprache rechts nicht gehört. Kein Schwindel.

In den obigen Krankengeschichten sind ausschliesslich Fälle erwähnt, in denen nur der horizontale Bogengang erkrankt war bezw. verletzt wurde. Die hierbei gemachten Erfahrungen decken sich im Ganzen mit denen früherer Jahre. In erster Linie sind die in den Vordergrund tretenden Symptome Schwindelerscheinungen, Nystagmus und subjective Gehörsempfindungen. Diese Symptome bestehen bei Erkrankungen des Bogengangs oft bereits vor der Operation, so dass man ohne Weiteres die Diagnose einer Erkrankung des Bogengangsapparates zu stellen in der Lage ist, oft aber auch fehlen sie und werden erst durch die Operation deutlich. Auffallend ist es, dass bei Kindern die Erscheinungen sowohl vorher als nachher fast gar nicht zu Tage treten.

Die Schwindelerscheinungen sind theils subjectiver Natur, theils lassen sie sich objectiv nachweisen.

Kurz nach der Operation, wenn die Kranken sich einigermaassen von der Chloroformnarkose erholt haben, klagen sie über Schwindelgefühl und Flimmern vor den Augen, die Gegenstände in Zimmer drehen sich, sie können nicht unterscheiden was unten und was oben ist. Legt man sie auf die Seite des kranken Ohres, so haben sie das Geftihl, als versänken sie im Bett. Beim Aufriehten werden dic Ersoheinungen heftiger, ebenso bei Bewegungen des Kopfes. Die meisten Kranken geben an, dass sich die Gegenstände im Zimmer nach einer Seite und zwar meist von der kranken zur gesunden hin drehten. Beim Blick auf die Strasse waren viele nicht im Stande, grosse Namen auf Firmenschildern zu lesen, die Buchstaben verwirrten sich. Bei längerem Blick auf die belebte Strasse verschoben und verzerrten sich die Bilder. Die Kranken selbst bekamen das Gefühl lebhaften Schwindels und konnten nicht mehr hinsehen. Bei geschlossenen Augen und im Dunkeln bestand starkes Sehwindelgefuhl. Auch Morgens beim Aufwachen und beim Versuch aus dem Bett aufzustehen war das Sehwindelgefühl stets stärker als am Tage. Auch das Aufstehen früh Morgens war stets von mehr Schwindelgefühl begleitet, als wie wenn der Kranke noch längere Zeit im Bett gelegen hatte und dann aufstand. Alle diese Symptome, beim einen stärker, beim anderen weniger stark anftretend, nahmen im Laufe der Zeit an Intensität $a b$ und verschwanden 
ganz. Bei 2 der Kranken (Fall III und IV) hielten die subjectiven Schwindelerscheinungen ausserordentlich lange an. Bei Fall IV, einem sonst sehr intelligenten Manne, zeigt sich ein Symptom besonders stark ausgeprägt. Noch 8 Monate nach der Operation machen ihn Gegenstände, die in Bewegung auf ihn zukommen, verwirrt und unsicher. Auf der belebten Strasse geht er, um mit ihm entgegenkommenden Mensehen nicht zusammen zu stossen, dicht an den Hüusern entlang. Ebenso "verwirren" ihn, schnell in den Bahnhof einfahrende Eisenbahnzüge. Bei einer solchen Gelegenheit wurde er, in der Absicht, sich einen Wagenabtheil zu sichern, den einfahrenden $\mathrm{Zug}$ fest fixirend, so schwindlig, dass er hinfiel. Dabei verlor er das Bewusstsein nicht.

Beim Verbandwechsel empfanden einige Kranke bei Reizung des Bogengangs durch Beriuhrung mit der Sonde lebhaftes Sohwindelgefühl.

Die in vielfachen Thierversuchen beobachteten, z. Th. planlosen z. Th. bestimmten Gesetzen folgenden, zwangsmässigen Bewegungen sowohl des Kopfes als auch der Extremitäten wurden auch andeutungsweise nie beobachtet.

Eine Verletzung bezw. Erkrankung des Kleinhirns oder des Grosshirns bestand bei keinem der Kranken und doch zeigten sich anch auffallende objective Schwindelerscheinnngen.

Fordert man einige Stunden nach der Operation die Kranken auf, den Kopf hochzuheben, so sind sie meist nicht dazu im Stande. Das ist insofern nicht auffällig, als es sich sehr oft bei Radicaloperirten findet, wohl als durch die Operation bedingte Folgeerscheinung von Druck- und Circulationsveränderungen im ganzen Labyrinth.

Hebt man aber den Kopf hoch, oder richtet man den Kranken auf, so kann der Kopf nicht sicher gehalten werden, oft fällt der Kranke sofort hintenuber unter lebhaftem Schwindelgefull. Lässt man nach einigen Tagen den Kranken im Bett sich aufsetzen, so kann er das oft zunächst allein nicht ausführen oder der Kranke schwankt hin und her und fällt schliessliob nach der Seite des operirten Ohres hin. Versucht man 5-6 Tage nach der Operation den Kranken hinzustellen, so fällt er sofort nach der erkrankten Seite hin um unter lebhaften Bewegungen der Arme, die offenbar dazu dienen sollen, ihn im Gleichgewicht zu erhalten. Bei einigen ist diese Erscheinung nicht so dentlich ausgeprägt. Am auffallendsten zeigt sie sich bei unbeabsichtigter 
Verletzung des Bogengangs (Fall I), oder bei operativ-nothwendiger grosser Zerstörung (Fall IV).

Charakteristisch ist die Art des Umfallens im Gegensatz zu solchen Kranken mit Erkrankung des Kleinhirns z. B. Hirnabscess. Während Kranke mit Verletzung des Bogengangs versuchen durch allerlei Bewegungen das Körpergleichgewicht aufrecht zu erbalten und sich ihrer Hilflosigkeit bewusst sind, sieht man, dass Kranke mit z. B. Hirnabscess selbst bei noch erhaltenem Bewusstsein hilflos in sich zusammensinken.

Sind nach weiteren Tagen (6. und 7. Tag nach der Operation) die Kranken im Stande zu gehen, so zeigt sich, dass sie entweder nur mit Unterstitzung gehen können, oder dass ihr Gang äusserst vorsichtig, langsam und breitbeinig ist. Hierbei ist zu bemerken; dass auch diese Erscheinungen der ersten Tage graduell sehr verschieden gefundeu werden.

Beim Gehen auf einen bestimmten Punkt hin zeigt sich eine gewisse Neigung, nach der erkrankten Seite abzuweichen. Es markirt sich dies auch schon an gezwungenen Armbewegungen, aus denen hervorgeht, dass die Kranken sich bewusst sind eine falsche Richtung einzuschlagen und versuchen durch Hilfsbewegungen dieselbe zu corrigiren. Bei Kehrtwendungen tritt auffallend starkes Schwanken ein, besonders bei Wendungen nach der kranken Seite. Uebersteigen eines Hindernisses füht zu unsicheren Bewegungen, ebenso Aufsteigen auf einen Stuhl. Unsicherer noch werden die Bewegungen im Dunkeln oder bei geschlossenen Augen, ebenso auch bei abnormer Kopfhaltung. Giebt man dagegen den Kranken eine leichte Unterstïtzung durch die Hand, so sind diese Schwindelbewegungen weniger deutlich ausgeprägt selbst im Dunkeln. Fehlt die Unterstützung, so sieht man daran, dass die Kranken mit gesenktem Blick Halt am Boden suchen, die Augen als Ersatzorgane auftreten.

Die beim Versuch, das Gleichgewicht zu erhalten, ausgeführten Bewegungen sind nicht planlos, sondern zweekgemäss. Der Gang ist im Gegensatz zu Kranken mit cerebellarer oder tabischer Ataxie nur vorsichtig breitbeinig, ohne unmotivirtes Schlenkern und Schleudern der Beine. Unregelmässige Bewegungen der Extremitäten werden nicht beobachtet. Herabsetzung der Sensibilität und Motilität ist nicht nachweisbar. In einem Falle (I) zeigte sich eine geringe Herabsetzung der Sensibilität der erkrankten Seite, für die eine bestimmte Ursache sich nicht nachweisen liess. 
Auch die objectiven Schwindelsymptome bilden sich im Verlaufe von Wochen und Monaten zurtuck. Eine ungefähr bestimmte Zeit lässt sich nicht angeben, meist sind es etwa 3 bis 4 Monate.

In fast allen Fällen von Erkrankung des horizontalen Bogengangs zeigt sich Nystagmus und zwar besteht derselbe meist als horizontaler in vielen Fällen vor der Operation, nach derselben fast ausnabmslos. Er tritt unmittelbar nach der Operation auf und verliert sich in $4-6$ Wochen, in einigen Fällen dauert er Monate lang. Meist zeigt er sich als einseitiger Nystagmus nach der ohrgesunden Seite hin, oder stärker nach dieser Seite bei beiderseitigem Nystagmus. Man unterscheidet immer deutlich eine langsam ausgeführte Bewegung nach der einen Seite und eine ruckartig ausgefuhrte nach der anderen Seite.

Eine fast ebenso regelmässig auftretende Erscheinung sind subjective Gehörsempfindungen. Dieselben werden oft ganz plötzlich in der 3 . bis 5 . Woche bemerkt, um sich nach verschieden langer Zeit 3-6 Wochen wieder zu verlieren. Sie sind ungleich stärker und quälender als die nach nncomplicirten Radicaloperationen in den ersten Wochen beobachteten subjectiven Gehörsempfindungen. Von den meisten Kranken wurden sie als laute, helle und schrille Geräusche geschildert, oft derart intensiv, dass sie den Schlaf störten. Ihre Entstehung ist wohl darauf zu schieben, dass im Verlaufe des Heilungsprocesses rom Bogengangscanal aus vielleicht durch narbige Veränderungen im Bereiche des Vorhofs vortibergehend Circulationsstörungen auftreten. Dass nach Operationen am Bogengang auffallend starkes und länger dauerndes Erbrechen sich einstellen sollte, lässt sich nach unseren Erfahrungen nicht bestätigen. In einzelnen Fällen trat es überhaupt nicht auf, in anderen war es nicht heftiger als sonst nach der Narkose. Er lässt sich auch diese Erscheinung mit der Funetion der Bogengänge nicht in Einklang bringen. Auch von Seiten der Kranken wurden keine besonderen Beschwerden über Uebelkeit oder bestehende Brechneigung geklagt.

Was das Gehör betrifft, so zeigt sich gegen das Resultat bei uncomplicirter Radicaloperation kein Unterschied. In einigen Fällen bleibt das an und für sich schlechte Hörvermögen unverändert, in einigen Fällen wird die Hörfähigkeit herabgesetzt, bleibt aber erhalten, in anderen Fällen wird das noch leidlich gute Hörvermögen nicht beeinflusst. 
Im Gegensatz zum Thierexperiment zeigen die Beobachtungen am Krankenbett, dass die bei Operationen am Bogengang auftretenden Gleichgewichtsstörungen und Bewegungen nicht zwangsmässig ausgeführte Bewegungen sind, sondern zweckmässig dazu dienen, das verlorene Gleichgewicht wieder herzustellen. Die Störungen, beim Thierexperiment $z$. Th. als Reizerscheinungen gedeutet, zeigen sich am Lebenden als typische Ausfallserseheinungen mit consecutiven Ersatzsymptomen. Der von Ewald als Ohrtonus beim Thierexperiment gedentete Symptomencomplex findet keine analogen Momente. Auch die Beobachtungen A dler's der den einseitigen Drehschwindel in einer Uebererregbarkeit des kranken Gleichgewichtsorganes begründet wissen will, lassen sich als z. Th. zu allgemeine zur Erklärung der Bogengangsfunction nicht verwerthen. Ganz unhaltbar aber ist die Lehre, den Bogengängen jede Spur einer statischen Function absprechen zu wollen. Unzweifelhaft auch lässt sich durch die Beobachtung. am Krankenbett nachweisen, dass die bei Bogengangsverletzungen bestehenden Sehwindelerseheinungen aufgetreten sind ohne irgend eine gleichzeitige Beschädigung des Gehirns. Wie liesse es sich anders erklären, dass bei einer einfachen Radicaloperation keine Schwindelerscheinungen anftreten, während bei einer gleichzeitigen Eröffnung des Bogengangs sofort die heftigsten Erscheinungen auftreten.

Wie anders wird die Beurtheilung der unter dem Bild der schweren Hysterie, Neurasthenie und Hypochondrie verlaufenden Fälle von Sohwindelerscheinungen sein, wenn wirklich die Ansicht sich bewahrheitet und durehgedrungen ist, dass der Bogengangsapparat ein Glied des Gleichgewichtsorgans ist, und dass der sogenannte Schwindel z. Th. anf einer organisehen Erkrankung eines bestimmten Organs beruht.

Unter den Erscheinungen des Schwindels bei Hysterie, Neurasthenie und Hypochondrie sind bei Hitzigi) Fälle beschrieben, von denen ich folgende anführen will.

B eobachtung I. K., 32 Jahre alt, Secretär.

Kopfverletzung durch Dachziegel auf die rechte Kopfseite; unmittelbar nachher Ohnmachtsanwandlung, so dass er $6-8$ Minuten gegen eine Thür gelehnt stehen bleiben musste; rinnenförmige Impression der hinteren oberen Ecke des rechten Scheitelbeins. Neben Schlaflosigkeit, Ohrensausen und Unfähigkeit, sich zu concentriren, hauptsächlich Klagen über Schwindel. Scheinbewegung des eigenen Körpers, so dass er beim Gehen nach rechts abzweigt, um einen scheinbaren Impuls nach links zu compensiren. Keine Scheinbewegung der Gesichtsobjecte. Flüsterworte links auf $6 \mathrm{~cm}$, werden rechts in

1) Der Schwindel. $1876-87$. 
$4 \mathrm{~cm}$ verstanden. Bei Augenfussschluss leichtes Schwanken, dabei wird der Kopf leicht nach links geneigt gehalten, während Patient die Empfindung hat, dass er ihn wegen der Tendenz, ihn nach links zu halten, thatsächlich mehr nach rechts hielt.

Beobachtung IV. P., 24 Jahre alt (5577).

Vor 9 Monaten Sturz von einem $12 \mathrm{~m}$ hohen Geräst, mehrere Wochen bewusstlos, Schwäche, Zittern, Vergesslichkeit, Sprachstörung, Pulsbeschleunigung, Narben auf dem Kopf. Schwindel beim Bücken und Aufwärtssehen, beim Schütteln des Kopfes; beim Stehen mit Angenfussschluss Umfallen, häufiges Taumeln beim Gehen und Bäcken, Gang breitbeinig, unsicher, mit kurzen Schritten. Beim Gehen mit geschlossenen Augen droht er sofort nach rechts umzufallen. Weicht beim Gehen beständig nach rechts ab. Es dreht sich Alles umgekehrt, wie der Uhrzeiger von rechts nach links, radförmig.

Beobachtung VIII. W., 42 Jahre alt (5799).

Vor $1^{1 / 2}$ Jahren Fall von der $2-3 \mathrm{~m}$ hohen Leiter auf den Kopf und die rechte Körperseite, dabei Kopfverietzung durch einen Spitzhammer; Bewusstlosigkeit. Neben anderen neurasthenischen Klagen Schwindelanfalle, bei denen es ihm schwarz vor den Augen, der Kopf schwer wird, als ob er ihn herunterfallt, muss sich dann auf den Stubl setzen oder sich festhalten; im Bette nie, wohl aber beim Gehen und auch beim Sitzen. Solche Anfälle mehrmals täglich ohne andere Veranlassung. Kann keine bestimmte Drehrichtung angeben. Beim Augenfussschluss kein Schwanken, dabei aber die kmpfindung, dass die rechte Körperhälfte schwerer sei, und als ob er den ganzen Körper mach links hinüber halten müsse, um nicht nach rechts zu fallen. Beim Blick nach oben, aussen, beim Bücken, beim Schütteln des Kopfs weder Schwindel, noch Schwanken, wohl aber die Empfindung der grösseren Schwere des Kopfes rechts.

Beobachtung XV. N., 49 Jahre alt (5985).

Fall von der stehenden Locomotive mit dem Räcken und der rechten Brustseite auf den Boden. Neben anderen neurasthenischen Erscheinungen hauptsächlich Klagen über Schwindel. Den ersten Anfall hatte er beim Ueberschreiten einer schmalen, nur auf einer Seite mit Geländer versehenen Brücke, er kam nur mit Mühe auf die andere Seite, seitdem wird er jedesmal schwindlig, wenn er sich rasch bewegende oder drehende Körper sieht, Carroussel, fliessendes Wasser, kann nicht am Coupéfenster sitzen. Beim Stehen mit geschlossenen Augen und Füssen ziemlich starkes Schwanken, so dass er umzufallen droht. Schwindelgefühl habe er dabei nicht, sondern nur Unsicherheit auf den Beinen. Längeres Fixiren beim Blick nach aussen ruft Schwindel und Schwanken im Sinne der Blickrichtung hervor, auch längeres Gehen ruft Schwindel hervor.

Vergleicht man diese Beobachtungen mit den nach Labyrinthverletzungen auftretenden Symptomen, so ist die Uebereinstimmung in die Augen fallend. Abweichen nach der einen Seite mit dem Bewusstsein, falseh zu gehen. Taumeln und Sohwanken, selbst Hinfallen bei offenen oder geschlossenen Augen. Breitbeiniger, unsicherer Gang mit kurzen Schritten. Astasie der Beinmuseulatur. Schwindelanfälle beim Betrachten bewegter Gegenstände. Heftigeres Auftreten der Schwindelanfälle Morgens nach dem Aufstehen. Diesen Fällen füge ich einen von mir in der letzten Zeit beobachteten an:

E. R., 61 Jahre alt, Kutscher. Fall 1897 auf Steinpflaster durch Bruch der Wagenachsen. 1 Stunde besinnungslos. Blutung aus Nase und rechtem Ohr. Behandlung wegen Sehädelbruchs. Nach dem Unfall Kopfschmerzen, Schwindel, Gedäehtnisssehwäche, 
schwankender Gang. 2 Jahre später Behandlung und Invaliditätserklärung wegen Unfallneurose. Jetzige Klagen Kopfschmerzen, besonders in der rechten Kopfseite. Gefühl von Pulsation rechts. Sehwindelgefubl, besonders auf der belebten Strasse, bei sehnellen Gehen und im Dunkeln. Befund: Bei Romberg Hin- und Herschwanken, nach einiger Zeit Schwanken nach rechts rïckwärts. Gang bei offenen Augen mit sehr kurzen Schritten, Augen fixiren den Boden. Abweichen nach rechts. Bei geschlossenen Augen anffallendes Schwanken nach rechts. Kehrtwendungen werden vorsichtig ausgeführt mit unregelmässigen Schwankungen. Stehen auf dem linken Bein möglich, auf dem rechten tritt lebhaftes Sohwanken ein. Patellarreflexe erhalten.

Nystagmus nach links.

Flüstersprache links $20 \mathrm{~cm}$ gehört, rechts nicht gehört. WL+. Es handelte sich hier unzweifelhaft um eine das Felsenbein durchsetzende Basisfractur, die nicht allein die Schnecke, sondern auch die Bogengänge getroffen hat. Hierfür spricht der Umstand, dass neben der durch die functionelle Ohruntersuchung gezeigten vollständigen Aufhebung des Hörvermögens bei der ersten Beobachtung eine rechtsseitige Facialisparese gefunden wurde, die sich auch jetzt noeh theilweise in den Mundpartien nachweisen lässt. Die subjectiven, sowic die objectiven Schwindelerscheinungen haben ihren Grund nicht in einer in Folge der Hirnerschütterung entstandenen allgemeinen Unfallneurose, sondern in der durch den Verlauf der Fissur naehgewiesenen Labyrinthverletzung.

Leider ist in den von Bitzig eitirten Fälen mit einer Ausnahme und auch hier nur andeutungsweise eine genauere vor Allem functionelle Ohruntersuchung nicht ausgeführt, wenigstens nicht erwähnt worden. Es lässt sich also nur aus der Art der Verletzungen auf eine Mitbetheiligung, Erschütterung, wenn nicht Bruch des Labyrinthes schliessen, wenigstens nur so lassen sich die im Gegensatz zu einer Hirnerschütterung immerhin auffallenden Erscheinungen erklären. Zur Beurtheilung von Schwindelanfällen müsste demnach jedesmal eine vor Allem functionelle Ohruntersuchung nothwendig sein.

Auf jeden Fall ist der Schwindel, wie wir ihn nach Bogengangsverletzungen beobachten, in seiner Art so bestimmt charakterisirt, wie wir es bei Hirntumoren, Hirnsyphilis, multipler Sklerose, Blutungen und Erweichungen, Epilepsie und Gerlierschen Sohwindel nicht beobachten und wenn der ibm ähnliche 
Symptomencomplex in der allgemeinen Bezeichnung des hysterischneurasthenisch-hypochondrischen Schwindels mit aufgegangen ist, so ist in jedem Falle festzustellen, in wie weit dies berechtigt ist.

Der nach Verletzung des Bogengangs auftretende Schwindel bildet sich nach einiger Zeit zurüek. Es müssen demnach, da das zerstörte Organ sich nicht wieder ersetzt, Ersatzorgane dessen Function übernehmen. Als solche kommen in Betracht das Auge, das Gehör, das Gleichgewichtsorgan der anderen Seite und der Muskelsinn. Was den Muskelsinn betrifft, so sehen wir, dass einige Kranke kurz nach der Bogengangsverletzung das Gefuhl hatten, als treten sie beim Gehen mit dem Bein der erkrankten Seite in ein Loch, andere hatten beim Liegen auf der kranken Seite das Gefuhl, als versänken sie im Bett. Ebenso weicht der Kranke beim Gehen auf einen bestimmten Punkt nach der kranken Seite $a b$, nicht weil auf dieser die Muskeln schwächer sind (wie man nach den $\mathrm{E}$ wald'schen Thierversuchen schliessen müsste), sondern weil das Gleichgewichtsgefïhl nach dieser Seite hin unsicherer ist. Der Kranke giebt diesem Gefubl dadurch Ausdruck, dass er durch Schlenkern mit den Armen und unsicheres Auftreten mit den Beinen, ,breitbeiniger Gang"6 sich im Gleichgewicht zu halten sucht. Nach einiger Zeit ist das Gefthl nicht mehr vorhanden. Es muissen sich demnach die Muskelgruppen an eine andere Controle gewöhnt haben, die wahrscheinlich von dem Labyrinth der anderen Seite ausgeht.

Auch das Auge muss entsehieden als Hilfsorgan zur Erhaltung des Gleichgewichts angesehen werden. Bei gesehlossenen Augen bezw. im Dunkeln sehen wir zunächst alle Erscheinungen des Schwindels lebhafter werden, die Kranken weichen von der vorgenommenen Richtung seitlich $\mathbf{a b}$, die Bewegungen werden unsicher und es treten Schwanken und auxiliäre Muskelbew egungen der Extremitäten ein. Auch das Bestehen des meist einseitigen Nystagmus bei Erkrankung des Bogengangs weist auf einen beiderseitigen Zusammenhang hin.

Dass anch das Hörlabyrinth als Hilfsorgan des Gleichgewichtsorgans aufzufassen ist, zeigt die Erscheinung, dass heftige Detonationen Schwindelgefühl erregen. Versuche bei Bogengangsverletzten duroh heftige Schalleinwirkungen Schwindelerscheinungen zu erregen, zeigten kein auffälliges Resultat.

In gewissem Grade unerklärlich ist die Beobachtung, dass bei Kindern Verletzung des Bogengangs so geringe Schwindelerscheinungen hervorruft. Vielleicht sind die Hilfsorgane des 
Gleichgewichtssinns noch nicht derart differenzirt, so dass das eine leichter für das ausfallende eintreten kann. So manpigfaltig auch die Hypothesen uber die Function der Bogengänge sind, Thierexperiment und Beobachtungen am Lebenden weisen gemeinsam in vielen Punkten darauf hin, dass der Bogengangsapparat ein Glied in der Kette der Organe ist, die den Gleichgewichtssinn darstellen. In wie weit die Bogengänge und Ampullen für sich oder der ganze Vorhof sich daran betheiligt, bedarf noch der weiteren Aufklärung. Die an Taubstummen gemachten Beobachtungen lassen sich insofern nicht zur Beurtheilung des Bogengangsschwindels verwerthen, als einerseits kein Werth darauf gelegt ist, in wie weit der Bogengangsapparat tuberhaupt ausgebildet und functionsfähig, anderseits in wie weit er erkrankt, resp. durch die Ersatzorgane in genügender Weise ersetzt ist.

\section{Literatur.}

1. Flourens, Recherches expérimentales sur les propriétés et les fonctions du septième nerf dans les animaux vertébrés. Paris 1842. -2 . Löwenberg, Ueber die nach Durchschneidung der Bogengănge des Ohrlabyrinths auftretenden Bewegungsstörungen. Arch. f. Ohrenheilk Bd. III. - 3. Goltz, Ueber die physiologische Bedeutung der Bogengänge des Ohrlabyrinths u. s. w. Pflüger's Archiv f. Physiol. Bd. IIf. - 4, M a ch, Grundlinien der Lehre von den Bewegungsempfindungen. Leipzig 1875. - 5. Breuer, Ueber die Function der liogengänge des Ohrlabyrinths. Med. Jahrb. 1874. - 6. Derselbe, Beiträge zur Lehre vom statischen Sinn. Ned. Jahrb. 1875. - Derselbe, Neue Versuche an den Ohrbogengängen. Pfüger's Arch. Bd. XLIV. - 6. Crum Brown, On the sense of Rotation etc. Proceding of the Royal Society of Ldingburg. Vol. VIII und Journ. of Anatomy and Physiol. VIII. - 7. Cy on, Bogengänge und Raumsinn. Archiv f. Anatomie u. Physiol. 1897. - 8. Laborde, Essai d'une détermination expérimentale et morphologique du rôle fonctionel des canaux semi-circulaires. Bulletin de la Societé d'anthropologie 1. déc. 1881. - 9. Viguiers, Le sens de l'orientation et ses organes chez les animaux et l'homme. Revue philosophique 1882. juillet. - 10. Delage, Ktudes expérimentelles sur ete. Archives de Zoologie expérimentale. T. IV. 1886. 11. E wald, Physiolog. Untersuchungen über das Endorgan des Nervus octarus. Wiesbaden 1891. - 12. Böttcher, Ueber die Durchschneidung der Bogengänge des Gehörlabyrinths und die sich daran knüpfenden Hypothesen. Arch. f. Ohrenheilk. Bd. IX. - 13. Berthold, Ueber die Function der Bogengänge des Ohrlabyrinths. Arch. f. Ohrenheilk. Bd. IX. - 14. Baginsky, Arch. f. Physiol. 1881 u. 1885 . - 15. v. Bergmann, Die Lehre von den Kopfrerletzungen. 1881. - 16. Curschmann, Archiv f. Physiol. Theil V. - 17. Brückner, Zur Function des Labyrinths. Virchow's Arch. CXIV. -. 18. Adler: Ueber den einseitigen Drehschwindel. Deutsche Zeitschr. f. Nervenheilkunde. Bd. XI. 